\title{
Evidence-based orthodontics: too many systematic reviews, too few trials
}

\author{
Papageorgiou, Spyridon N ; Eliades, Theodore
}

\begin{abstract}
Why are there so many systematic reviews in orthodontics, and what are the negative consequences of this for our speciality as an academic discipline?
\end{abstract}

DOI: https://doi.org/10.1177/1465312519842322

Posted at the Zurich Open Repository and Archive, University of Zurich ZORA URL: https://doi.org/10.5167/uzh-186125

Journal Article

Accepted Version

Originally published at:

Papageorgiou, Spyridon N; Eliades, Theodore (2019). Evidence-based orthodontics: too many systematic reviews, too few trials. Journal of Orthodontics, $46\left(1_{s}\right.$ uppl $): 9-12$.

DOI: https://doi.org/10.1177/1465312519842322 
Evidence-based orthodontics: too many systematic reviews, too few trials

Spyridon N. Papageorgiou ${ }^{1}$, Theodore Eliades ${ }^{1}$

${ }^{1}$ Clinic of Orthodontics and Pediatric Dentistry, Center of Dental Medicine, University of Zurich, Zurich, Switzerland

ORCID

Spyridon N. Papageorgiou: 0000-0003-1968-3326

Theodore Eliades: 0000-0003-2313-4979

CONTACT Spyridon N. Papageorgiou Clinic of Orthodontics and Pediatric Dentistry, Center of Dental Medicine, University of Zurich, Plattenstrasse 11, Zurich CH 8032, Switzerland; snpapage@gmail.com.

Words in text: 1597

\section{Disclosure statement}

No potential conflict of interest was reported by the author. 


\section{MANUSCRIPT}

\section{Introduction}

Under the principles of evidence-based medicine, clinical trials are the epitome of primary comparative research on humans and form the basis for deciding which treatment might work best for a specific patient problem. Randomisation of patients into groups to be compared is crucial in ensuring similarity of the groups for any known or unknown confounders, so that any difference seen at the trial's end can be directly attributed to the administered intervention. Allocation concealment and blinding of all entities involved in a trial (patients, orthodontists, and outcome assessors) helps minimising selection, performance, and detection bias and further enhances the robustness of the scientific procedure. Finally, pre-registration of the full trial protocol helps in having transparency throughout the research process and minimises the risk of publication bias by making trial traceable from the start. However, sometimes even well-designed clinical trials cannot always fully answer a clinical research hypothesis.

\section{Shortcomings of clinical trials}

Planning, conducting, and monitoring a clinical trial is an arduous task and considerable time, effort, and money is required to recruit, treat, and then follow the included patients throughout the trial. As a result, clinical trials often include much smaller patient samples than non-randomised studies, due to the increased costs. Indeed, such often called 'pilot' trials with very small sample sizes are unfortunately very prevalent in orthodontic literature and can include as little as 10 patients, while almost $20 \%$ of trials include less than 30 patients (re-analysed from Al-Mohrabi et al. 2018). This hampers the power of clinical trials to identify an existing difference between two treatments and affects the precision of their estimates. This has been illustrated by data indicating that initial small trials with statistically significant findings are later on refuted by subsequent larger trials (Cappelleri et al. 1996; loannidis, 2005). Furthermore, issues in the conduct or reporting of clinical trials can introduce bias or limit the useful information that can be extracted from a trial report (Bearn and Alharbi, 2015; Papageorgiou et al. 2015; Koletsi et al. 2016; Papageorgiou et al. 2018). Additionally, the results of each single clinical trial can be directly applicable only to populations similar to the patients included in the trial and might not be safely transferable to other patients. Generalisability of a trial's results can therefore only be attained by setting multiple trial centres that cover diverse patient groups 
or by synthesising multiple clinical trials. This enables to have a wider range of patients and clinical settings, thereby introducing the clinical variation that is seen in clinical reality.

\section{Evidence synthesis from multiple trials}

Synthesis of data from multiple clinical trials is properly done by performing meta-analyses within the framework of a systematic review of existing literature, as this enables a transparent and mostly objective format to identify and combine the entirety of existing primary research around a scientific question. The main strengths of meta-analysis include combining data on a treatment's clinical performance to a single estimate with increased statistical power, illustrating the clinical heterogeneity that might be expected in clinical situations, and assessing the influence of patient-, treatment-, or study-related characteristics on the treatment's performance. Sometimes meta-analyses might even be able to shed light on novel research questions that were not raised in the included original primary trials. They also provide balanced and transparent evidence for patients, relatives, and policymakers about the benefits / harms of interventions and they can be the starting point for trustworthy clinical practice guidelines (Guyatt et al 2008). However, systematic reviews and meta-analyses are a form of secondary research, which means they are encumbered by the existence of well-designed primary clinical trials to fuel evidence-based recommendations. Additionally, the credibility of a meta-analysis is dependent on both the scientific rigor (the 'risk of bias') of included primary trials and on the robustness of the systematic review / meta-analysis methods used to combine these trials (Papageorgiou 2014). This means that meta-analysis has potentially double the risk of bias compared to primary studies, while at the same time meta-analysis of flawed studies only leads to flawed or potentially misleading pooled estimates. In a way, meta-analysis might be compared to a wall or a building, whereas single trials are the single building blocks used to construct this. The stability

of the construct is only secured by solid building blocks that interlock well, whereas flawed primary trials lead to meta-analysis of questionable stability / credibility. Adding to that, meta-analyses rely on the reporting completeness of randomized trials' papers, which has been shown to be often problematic (Bearn and Alharbi, 2015) and can limit the meta-analyses. Overall, the quality of systematic reviews or metaanalyses might be hampered by many factors, including risk of bias, indirectness, imprecision, inconsistency, and publication bias, which can affect the validity of their findings (Guyatt et al 2008). 


\section{Published randomised trials and systematic reviews in orthodontics}

Notwithstanding this, systematic reviews and meta-analyses are becoming increasingly popular in orthodontic literature and lately more systematic reviews / meta-analyses than randomised trials are published each year (Table 1; Figure 1). This trend might be explained by many factors.

First, systematic reviews require on average less resources than clinical trials (Glasziou et al. 2006) and might be completed within a shorter timeframe-whereas a randomised trial of comprehensive orthodontic treatment might take many years. Second, systematic reviews might be on average 'cheaper' than clinical trials, but receive equal, if not more, citations than randomised trials and other study designs (Patsopoulos et al. 2005). Third, systematic reviews do not per se involve any procedures on human patients and do not require ethical clearance beforehand (Greene and Bearn 2013), which makes it a popular research project for postgraduate students or junior researchers. Finally, many journals or funding agencies ask that any new large clinical trial on a field should begin and end with a systematic review of existing literature to help put the trial's results into context (Clarke et al. 2010). In such cases, systematic reviews are justified as they help reduce research waste originating from unjustified research and might minimise burden or adverse effects in patients allocated to relatively less effective treatments in new trials (Chalmers et al. 2014).

There is however something amiss when disproportionately more secondary research is conducted on the expense of primary research. For one, treatment techniques and philosophies constantly evolve with increasingly accumulated evidence and experience, while novel materials and appliances are constantly marketed. If clinical trials do not keep up with recent developments, then researchers and clinicians alike run the risk of falling behind the times (Seehra et al. 2017), which might translate to novel treatments being used on patients without prior knowledge about their efficacy or safety. Additionally, recycling already existing clinical trials in new systematic reviews with overlapping meta-analyses provides no additional new information and can be regarded as research waste in terms of unnecessarily spent resources. Some overlap between meta-analyses might be desirable for either replication purposes or to correct the record set by an initially misleading meta-analysis due to biased use of eligibility criteria, outcome definitions, analyses, or interpretation of results. Also, updating the evidence provided by an older meta-analysis is another valid reason to perform a new meta-analysis on the subject (Garner et al. 2016), but should ideally be preceded by documented publication of a minimum number of new and meaningful clinical trials. 
Importantly, there may be less noble incentives for doing another redundant systematic review, as industrysponsored systematic reviews conducted by contractors might be used as a particularly powerful marketing tool (loannidis, 2016). Therefore, careful examination of sponsoring, conflicts of interests, and transparency of the report is a crucial, yet often overlooked, aspect of a study's critical appraisal.

\section{Summary and recommendations}

Systematic reviews and meta-analyses have the potential to play an important role in evidence-based decision-making by collating and appraising all existing clinical trials pertaining to a specific question. They need however to be both true and useful to clinicians and researchers alike, which means that reviewers should go at great lengths to minimise bias and research waste by. To this end, there are several steps that researchers or editors and peer-reviewers of scientific journals can take. As a minimum, researchers planning on undertaking a systematic review should have in-depth knowledge of the field they wish to cover and be aware of most primary studies and systematic reviews existing in the field. Prior to registering the protocol of their systematic review in PROSPERO (Sideri et al. 2017) the authors should ensure that the research question has not been previously addressed in another review or that an adequate number of trials have been published since the last review to justify its update. As far as proper methodology for systematic reviews or meta-analyses are concerned, detailed guidance can be found in the Cochrane Handbook for Systematic Reviews of Interventions (Higgins and Green, 2011). Furthermore, as with any type of research, transparency in terms of both sponsorship or conflicts of interest, as well as open data sharing (Papageorgiou and Cobourne, 2018) is of paramount importance in building trust on systematic reviews. Finally, peer-reviewers and editors of orthodontic journals need to be aware of the massive production of systematic reviews and ensure that these are indeed carefully conducted and useful additions to the existing

literature. Just as a novel badly-conducted systematic review of previously unassessed trials might mislead readers, so is a well-designed duplicate systematic review of previously covered trials equally unfit for publication. Critical appraisal and watchful monitoring of the systematic reviews that manage to get publish will be useful in both improving the quality of existing evidence in orthodontics and - hopefully - encourage the conduct of additional well-designed clinical trials. 


\section{References}

Al-Moghrabi D, Tsichlaki A, Pandis N, Fleming PS. Collaboration in orthodontic clinical trials: prevalence and association with sample size and funding. Prog Orthod. 2018 Jun 11;19(1):16.

Cappelleri JC, loannidis JP, Schmid CH, de Ferranti SD, Aubert M, Chalmers TC, Lau J. Large trials vs meta-analysis of smaller trials: how do their results compare? JAMA. 1996 Oct 23-30;276(16):1332-8. loannidis JP. The Mass Production of Redundant, Misleading, and Conflicted Systematic Reviews and Meta-analyses. Milbank Q. 2016 Sep;94(3):485-514.

Bearn DR, Alharbi F. Reporting of clinical trials in the orthodontic literature from 2008 to 2012: observational study of published reports in four major journals. J Orthod. 2015 Sep;42(3):186-91.

Chalmers I, Bracken MB, Djulbegovic B, Garattini S, Grant J, Gülmezoglu AM, Howells DW, loannidis JP, Oliver S. How to increase value and reduce waste when research priorities are set. Lancet. 2014 Jan 11;383(9912):156-65.

Clarke M, Hopewell S, Chalmers I. Clinical trials should begin and end with systematic reviews of relevant evidence: 12 years and waiting. Lancet. 2010 Jul 3;376(9734):20-1.

Garner P, Hopewell S, Chandler J, MacLehose H, Schünemann HJ, Akl EA, Beyene J, Chang S, Churchill R, Dearness K, Guyatt G, Lefebvre C, Liles B, Marshall R, Martínez García L, Mavergames C, Nasser M, Qaseem A, Sampson M, Soares-Weiser K, Takwoingi Y, Thabane L, Trivella M, Tugwell P, Welsh E, Wilson EC, Schünemann HJ; Panel for updating guidance for systematic reviews (PUGs). When and how to update systematic reviews: consensus and checklist. BMJ. 2016 Jul 20;354:i3507.

Glasziou P, Djulbegovic B, Burls A. Are systematic reviews more cost-effective than randomised trials? Lancet. 2006 Jun 24;367(9528):2057-8.

Greene LE, Bearn DR. Setting up a randomized clinical trial in the UK: approvals and process. J Orthod. 2013 Jun;40(2):104-11

Guyatt GH, Oxman AD, Vist GE, Kunz R, Falck-Ytter Y, Alonso-Coello P, Schunemann HJ (2008) GRADE: an emerging consensus on rating quality of evidence and strength of recommendations. BMJ 336:924926.

Higgins JPT, Green S. 2011. Cochrane handbook for systematic reviews of interventions 5.1 .0 (updated 2011 Mar); [accessed 2018 February 5]. http://handbook-5-1.cochrane.org/ 
loannidis JP. Contradicted and initially stronger effects in highly cited clinical research. JAMA. 2005 Jul 13;294(2):218-28.

Koletsi D, Spineli LM, Lempesi E, Pandis N. Risk of bias and magnitude of effect in orthodontic randomized controlled trials: a meta-epidemiological review. Eur J Orthod. 2016 Jun;38(3):308-12.

Papageorgiou SN, Cobourne MT. Data sharing in orthodontic research. J Orthod. 2018 Mar;45(1):1-3.

Papageorgiou SN, Xavier GM, Cobourne MT, Eliades T. Registered trials report less beneficial treatment effects than unregistered ones: a meta-epidemiological study in orthodontics. J Clin Epidemiol. 2018 Aug;100:44-52.

Papageorgiou SN, Xavier GM, Cobourne MT. Basic study design influences the results of orthodontic clinical investigations. J Clin Epidemiol. 2015 Dec;68(12):1512-22.

Papageorgiou SN. Meta-analysis for orthodontists: Part II--Is all that glitters gold? J Orthod. 2014 Dec;41(4):327-36.

Patsopoulos NA, Analatos AA, loannidis JP. Relative citation impact of various study designs in the health sciences. JAMA. 2005 May 18;293(19):2362-6.

Seehra J, Pandis N, Fleming PS. Clinical evaluation of marketed orthodontic products: are researchers behind the times? A meta-epidemiological study. Prog Orthod. 2017 Dec;18(1):14.

Sideri S, Papageorgiou SN, Eliades T. Are orthodontic systematic reviews registered a priori in PROSPERO? J Orthod. 2017 Dec;44(4):249-255. 
Figure 1. \% part of total publications in each year comprising of Randomised Clinical Trials (RCTs) or Systematic Reviews / Meta-Analyses (SRs/Mas). Data exported using the corresponding filters in MEDLINE via Pubmed on January 15 2019 (https://doi.org/xyzxyz/zenodo.xyzxyz).

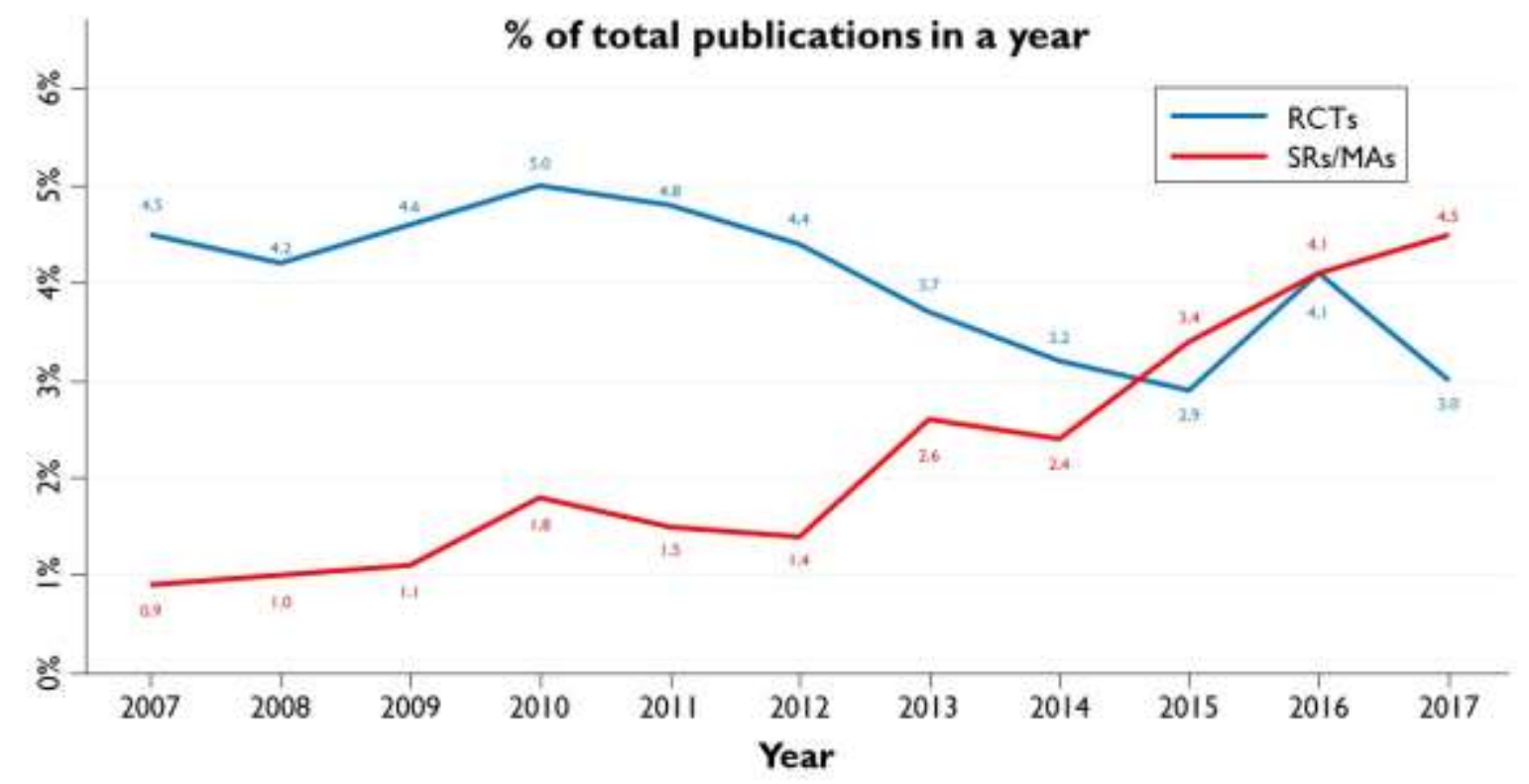


Table 1. Number of publications listed in MEDLINE (through PubMed) each year using the simple search orthodont ${ }^{*}$ and selecting PubMed's filters for randomised trials or systematic reviews / meta-analyses.

\begin{tabular}{|l|l|l|l|l|l|l|}
\hline & & \multicolumn{2}{|l|}{ RCTs } & \multicolumn{2}{|l|}{ SRs/MAs } \\
\hline Year & $\begin{array}{l}\text { Total } \\
\text { publications }\end{array}$ & Number & $\begin{array}{l}\% \text { of } \\
\text { total }\end{array}$ & Number & $\begin{array}{l}\% \text { of } \\
\text { total }\end{array}$ \\
\hline 2007 & 1724 & 78 & $4.5 \%$ & 16 & $0.9 \%$ \\
\hline 2008 & 1855 & 78 & $4.2 \%$ & 19 & $1.0 \%$ \\
\hline 2009 & 1919 & 88 & $4.6 \%$ & 21 & $1.1 \%$ \\
\hline 2010 & 2036 & 101 & $5.0 \%$ & 36 & $1.8 \%$ \\
\hline 2011 & 2122 & 102 & $4.8 \%$ & 32 & $1.5 \%$ \\
\hline 2012 & 2434 & 108 & $4.4 \%$ & 35 & $1.4 \%$ \\
\hline 2013 & 2615 & 97 & $3.7 \%$ & 67 & $2.6 \%$ \\
\hline 2014 & 3079 & 99 & $3.2 \%$ & 74 & $2.4 \%$ \\
\hline 2015 & 3466 & 102 & $2.9 \%$ & 119 & $3.4 \%$ \\
\hline 2016 & 3302 & 136 & $4.1 \%$ & 134 & $4.1 \%$ \\
\hline 2017 & 3307 & 99 & $3.0 \%$ & 149 & $4.5 \%$ \\
\hline
\end{tabular}

MA, meta-analysis; RCT, randomised clinical trial; SR, systematic review. 